\title{
Non-linear electro-optic modelling of a Barium Titanate grating coupler
}

Croes, Guillaume, Smolentsev, Nikolay, Wang, Tsang Hsuan, Gehlhaar, Robert, Genoe, Jan

Guillaume Croes, Nikolay Smolentsev, Tsang Hsuan Wang, Robert Gehlhaar, Jan Genoe, "Non-linear electro-optic modelling of a Barium Titanate grating coupler," Proc. SPIE 11484, Optical Modeling and Performance Predictions XI, 114840D (20 August 2020); doi: 10.1117/12.2568032

SPIE. Event: SPIE Optical Engineering + Applications, 2020, Online Only 


\title{
Non-linear electro-optic modelling of a Barium Titanate grating coupler
}

\author{
Guillaume Croes $^{\mathrm{a}, \mathrm{b}}$, Nikolay Smolentsev ${ }^{\mathrm{a}, \mathrm{b}}$, Tsang Hsuan Wang ${ }^{\mathrm{a}, \mathrm{b}}$, Robert Gehlhaar ${ }^{\mathrm{a}}$ and Jan \\ Genoe $^{\mathrm{a}, \mathrm{b}}$ \\ ${ }^{a}$ Imec, Kapeldreef 75, 3001, Leuven, Belgium; ${ }^{b}$ Electrical Engineering Dept. (ESAT), Katholieke \\ Universiteit Leuven, 3001 Leuven, Belgium
}

\begin{abstract}
We provide insight into the driving mechanisms and requirements to create an electro-optic spatial light modulator based on a Barium Titanate waveguide and an optically transparent electrode cladding layer. We have developed a generic framework of electric field simulations and non-linear optics to create any desired modulation in an area of interest, applicable for liquid crystals, Pockels and Kerr cells. Targeting our device structure, we have evaluated several design parameters of the arbitrarily reprogrammable SLM, capable of optical beamforming and high-quality holograms.
\end{abstract}

Keywords: Beam Steering, Electro-Optic effect, Grating Coupler, Barium Titanate

\section{INTRODUCTION}

Over the years, a wide range of technologies have emerged aspiring to realize advanced beam steering topologies. ${ }^{1,2,3}$ Some of the more important ones include small feature sizes, rapid switching and scalability. The feature sizes determine the accuracy and range of the obtained beam angle. Ideally, subwavelength sized features are created as this enables a viewing angle of $180^{\circ} .4,5$ The current state-of-the-art technologies that have shown most promise are liquid crystals, microelectro-mechanical systems, surface acousto-optic modulators and phase-change materials. ${ }^{6,7,8,1,2,3}$ All offer a degree of switchability while slightly lacking in feature sizes leading to rather small viewing angles and some optical artefacts. This naturally limits the flexibility of these technologies as they require additional components for compensation. Artefact prevention with low complexity systems is one of the main challenges in this field.

Of all these state-of-the-art technologies, it was a surface acousto-optic modulator that has probably come closest to meeting the requirements highlighted above. This is due to its design having some inherent advantages over the other technologies. For example, by using an anisotropic waveguide a mode coupler can be created showing polarization rotation between guided and radiation modes, leading to reduced noise. We want to expand on this concept and try to improve it further by applying electro-optic (EO) modulation instead. Our envisioned device resembles an electro-optically modulated long period grating meant to control transmission spectra. ${ }^{9,10,11}$ In contrast with these works, we do not employ counter electrodes on top of the slab waveguide. Naturally, these electrodes can generate more homogeneous electric fields and thus result in an improved refractive index modulation within the waveguide. However, counter electrodes serve as an extra source of attenuation for the guided and outcoupled light.

The spatial light modulator presented here consists of a planar EO slab waveguide that is bonded to a transparent cladding electrode array. Each neighboring electrode pair is able to modulate the refractive index in the corresponding waveguide volume. Careful tuning of each pixel allows the construction of an entire refractive index modulated slab designed to partially couple out waveguided light. The versatile design can be used for beam steering, filtering, mode conversion and eventually also holography. We describe the operation and design constraints of this device, focusing on a two-dimensional version to keep the required parameters limited. Our work is centered around barium titanate (BTO) but the formalism laid out here can also be extended to liquid crystal cells or any other electro-optic material.

The $100 \mathrm{~nm}$ electrode pitch enables applying any relevant periodic structure for light in the visible range and hence also the quick switching between different patterns.

As stated above, the main component of the envisioned device is an EO slab waveguide. A simplified schematic of the structure is shown in Figure 1, where the xz plane of the device is shown. In the 2D scope presented here a slab waveguide and infinitely long electrodes perpendicular to the propagation direction can be used. To ensure the highest possible control over the waveguide, BTO was chosen as it has one of the strongest EO responses. ${ }^{12}$ The transparent conductor in the 
cladding layer brings the electrodes close to the waveguide while simultaneously minimizing any undesired effects such as absorption or scattering that arise when a metal electrode would be used. Below these electrodes are titanium nitride (TiN) contacts that form the connection to a silicon backplane.

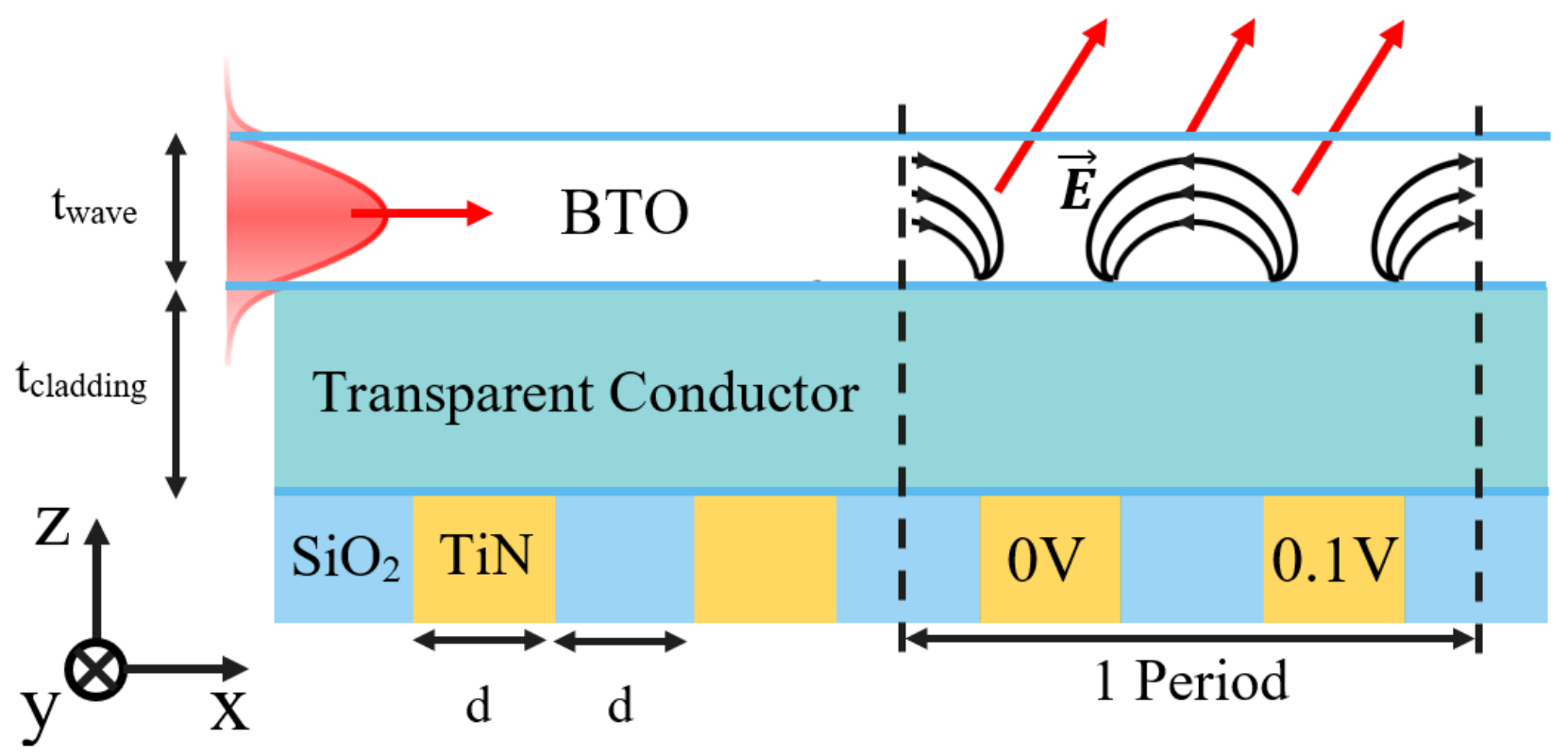

Figure 1. Schematic of the electro-optically modulated waveguide build on a conductive cladding.

In practice, the device operates as a precise mode coupler employing the anisotropic nature of the BTO waveguide. In the scenario presented here, we will focus on TE guided modes coupled to TM radiation modes through the electro-optic effect. Within this work, we focus on a device operating at a wavelength $\lambda=550 \mathrm{~nm}$ and will adjust the other parameters to suite this wavelength. The employed waveguide and conductive transparent cladding thicknesses, indicated with $t_{\text {wave }}$ and $\mathrm{t}_{\text {cladding }}$ in Figure 1, are $200 \mathrm{~nm}$ and $500 \mathrm{~nm}$ respectively. To ensure proper control over the waveguide and minimize higher grating orders, the electrodes are chosen to have a size $\mathrm{d}$ of $50 \mathrm{~nm}$. That said, the method we present can be completely generalized to suite any grating coupler application.

This paper will first focus on the potentials and accompanying electric fields that the electrode array can apply to the waveguide. A COMSOL simulation is introduced to calculate the electric field applied by an infinite series of electrodes that are biased periodically. Here we will also highlight the origin of the extreme flexibility of our method as practically any desired electric field profile can be created in the waveguided using a similar method. In Section 3, we briefly touch on the electro-optic response from the waveguide and discuss the influence of the crystalline phases of BTO. Next, we present a theoretical approach to assess the coupling strength of the calculated refractive index modulation. Finally, we simulate our mode coupler by performing finite difference time domain (FDTD) simulations.

\section{ELECTRIC FIELD SIMULATION}

A stationary electrostatic finite element simulation was set up to calculate the desired electric field by using COMSOL. The schematic of the simulated period is outlined in Figure 1. The electrodes were constructed by applying a terminal boundary condition to their edges as their inside does not contribute to the electric field generation. The rest of the boundaries, except for the period edges, were left at the standard zero charge condition. The relative permittivities $\varepsilon_{r, B T O}$, $\varepsilon_{r, S i N}$ of BTO and SiN were chosen to be $(3600,150,3600)$ and 7 respectively. ${ }^{13,14,15,16}$ This phase of BTO corresponds to an a-oriented crystal of which the extra-ordinary axis lies out of plane, this specific choice will be detailed when the electrooptic response of the waveguide is discussed. Of the two electrodes inside the simulated period, one was grounded whilst the other was biased at $0.1 \mathrm{~V}$. Since higher voltages brought the electric field strength close to the dielectric breakdown of the employed materials, we chose this as a cautionary option. The electric field and potential data were exported on a linearly spaced grid with intervals of $1 \mathrm{~nm}$ to create a 200 by 200 sized matrix. It must be noted that this is the simplest possible grating that can be applied, by varying the potentials and the period various kinds of grating shapes, such as 
slanted or chirped gratings can be created. This flexibility makes this device such an interesting application in the beam forming field.

The results of the COMSOL simulation illustrate the electric field distribution with the highest field strength close to the waveguide-cladding interface (Fig. 2a). As a result, most of the coupling will originate from this area. The separate electric field components, namely $E_{x}$ and $E_{z}$ are rather similar in absolute magnitude, aligning with the values of the relative permittivity (Fig.2b and c). Interestingly, they appear translated by $50 \mathrm{~nm}$ from each other. Additionally, the graphs depict the period of the constructed waveguide grating. Even though the individual electrodes have a period of $100 \mathrm{~nm}$, the resulting grating has a doubled period due to the negative and positive electric fields.

\section{ELECTRO-OPTIC RESPONSE}

The electro-optic response of the BTO waveguide was assessed by applying the nonlinear permeability tensor $\eta_{i j}$. In the case of an anisotropic material under influence of an external electric field, this tensor has a more general form than for an isotropic material. Usually it is written in a series notation where each term represents a part of the electro-optic effect depending on how it scales with the applied electric field.

$$
\eta_{i j}=\eta_{i j}^{(0)}+\sum_{k} r_{i j k} E_{k}+\sum_{k l} s_{i j k l} E_{k} E_{l}+\ldots
$$
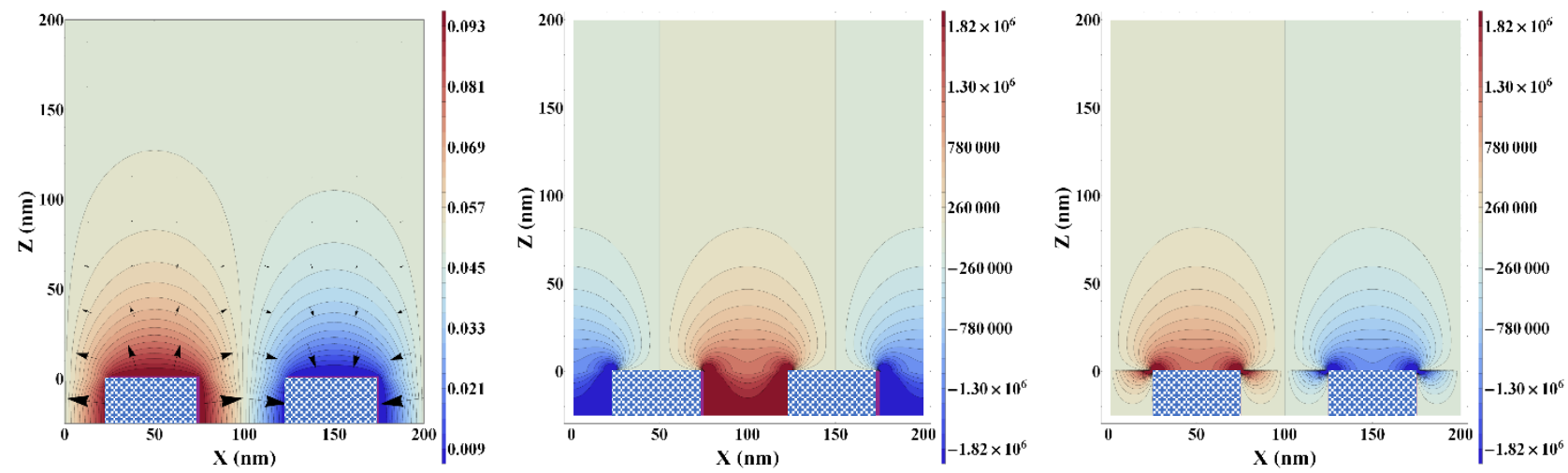

Figure 2: a) A contour plot of the potential given by a single period of the COMSOL simulation. Electric field vectors with their size linked to the electric field strength are indicated by the black arrows. b) A contour plot of the electric field component along the $\mathrm{x}$-axis. c) Contour plot of the electric field component along the z-axis.

Here, the linear electro-optic effect expressed by the Pockels coefficients $r_{i j k}$, will be used for modulating the refractive index in the waveguide. In the case of c-oriented BTO, with its extraordinary axis lying along the z-axis, the perturbed permeability tensor takes on the following shape. ${ }^{19}$

$$
\eta_{i j}{ }^{\prime}=1 / n^{\prime 2}=1 / \varepsilon_{i j}{ }^{\prime}=\left[\begin{array}{ccc}
\frac{1}{n_{x}{ }^{2}}+r_{13} E_{z} & 0 & r_{42} E_{y} \\
0 & \frac{1}{n_{x}{ }^{2}}+r_{13} E_{z} & r_{42} E_{x} \\
r_{42} E_{y} & r_{42} E_{x} & \frac{1}{n_{z}{ }^{2}}+r_{33} E_{z}
\end{array}\right]
$$

Where $n_{x}=2.488$ and $n_{z}=2.424$ are the refractive indices along the $\mathrm{x}$ and $\mathrm{z}$ axis. The Pockels coefficients are equal to $r_{13}=19.5 \mathrm{e}-12 \mathrm{~m} / \mathrm{V}, r_{33}=97 \mathrm{e}-12 \mathrm{~m} / \mathrm{V}$ and $r_{42}=1640 \mathrm{e}-12 \mathrm{~m} / \mathrm{V}$. For the other phases of BTO, we believe it is more intuitive to stick to this tensor form and use a coordinate transformation. To then fully understand how the Pockels effect has modulated the BTO, this tensor needs to by diagonalized and its eigenvectors extracted, such that any form of stretching, compression and rotation of the index ellipsoid is accounted for. To our advantage, the coupled mode theory directly uses the permittivity tensor instead of the refractive indices. 
After looking at the electric field simulation and the accompanying modulated permittivity tensor of each of the BTO phases, a-oriented BTO with its extraordinary axis lying out of plane was selected for further investigation. In part, because the magnitude of the Pockels coefficients used by the relevant tensor elements, but also because of the present electric field. The electric fields for the other BTO phases, having the smallest $\varepsilon_{r}$ component along the $\mathrm{x}$ or $\mathrm{z}$ axis, were either localized to the waveguide-cladding interface or stretched very deep into the BTO.

Since the goal was to couple a TE guided mode to a TM radiation mode the change of the permittivity matrix elements is of interest. It can be found by taking the inverse of Eq. 2 and subtracting the permittivity under normal circumstances. In the end, most of the tensor elements do not contribute according to coupled mode theory. In the employed coordinate system, the guided mode only has an $E_{y}$ component, whereas the TM radiation mode has both an $E_{x}$ component and $E_{z}$ component. Looking back at the permittivity tensor, only two elements can thus be used for coupling namely $\Delta \varepsilon_{y x}$ and $\Delta \varepsilon_{y z}$. Both components are shown in Figure 3. In absolute size, element $\Delta \varepsilon_{y z}$ completely overshadows $\Delta \varepsilon_{y x}$ suggesting strong coupling between the $E_{y}$ guided field and the $E_{z}$ radiation field.

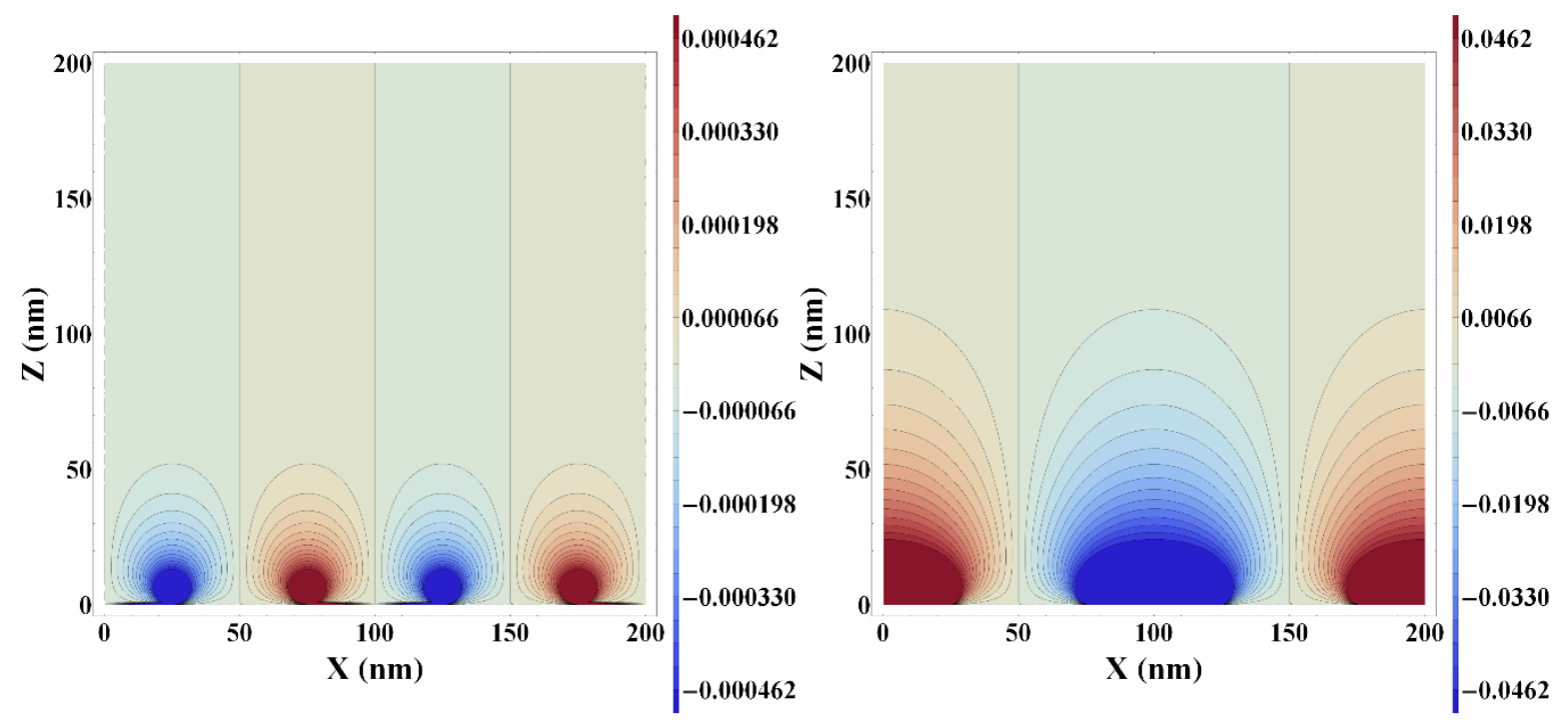

Figure 3: a) A contour plot of the perturbation the electric field creates for the $\Delta \varepsilon_{y x}$ element of the permittivity tensor. b) A contour plot of the $\Delta \varepsilon_{y z}$ element perturbation of the permittivity tensor.

\section{THEORETICAL EXPECTATION}

Coupled mode theory is used to analytically model the grating behavior. By simplifying the internal electric field distribution, it is a fast approximative method used in parallel with the finite difference time domain (FDTD) simulations of Section 5 and thus can be used a validation for their results. Previous works have already applied coupled mode theory to a slab waveguide modulated by a homogeneous cosine shaped grating. ${ }^{20}$ Here, we extend this theory by generalizing the kind of grating that can be assessed, specifically looking at any possible grating shape and gratings that vary in strength throughout the waveguide.

In general, we will follow S.M Norton's work and will highlight here where we deviate from his approach. A good starting point for our method are the coupling coefficients between the guided and radiation modes which are typically split up in transverse and longitudinal components called $K^{t}$ and $K^{x}$ respectively. ${ }^{21,22}$ In the case of our TE guided mode, no longitudinal electric field is present meaning that only $K^{t}$ will play a role here. For $K^{t}$ we can then write.

$$
K^{t}{ }_{v: q \rho}=\frac{\omega}{4} \int_{-\infty}^{\infty} \Delta \varepsilon_{i j}(x, z) \boldsymbol{E}_{t v} \cdot \boldsymbol{E}_{q \rho} d z
$$

Here we assume a forward propagating bound mode along the $\mathrm{z}$ axis denoted with $v$ and a continuous spectrum of radiation modes denoted with $\rho, q$ indicates of the modes are symmetric or antisymmetric. 
Important here is that we use a different guided mode than in the work mentioned above, instead of an analytical approach a numerical solver (LUMERICAL Modesolver) is used. This is needed to factor in the underlying metal contacts and silicon backplane as the simplified slab waveguide approach cannot handle the detailed geometric structure we implement. Consequently, the result is a set of sampled values coinciding with the employed modesolver mesh. A similar result is found in the COMSOL simulation where the electric field and the accompanying permittivity depend on the mesh precision. Both datasets prompt us to step away from the integral and use a Riemann sum over the waveguide thickness instead. This same exercise can be made along the entire length of the periodic structure resulting in a dataset of coupling coefficients each having a different $\mathrm{x}$ coordinate.

The outcome of the Riemann sum, although periodic, is by no means resembling of a cosine. As such, the theory this work is based on needs to be expanded on. To use it to its full extend a cosine series expansion of the coupling coefficient dataset was taken of which the individual terms can subsequently be assessed separately. A Fourier discrete cosine transform was used to see what spatial frequency components were present. The employed series is the following where is $n$ is the number of elements in the dataset.

$$
K^{t}(x)=\frac{1}{\sqrt{n}}\left(u_{1}+2 \sum_{r=2}^{n} u_{r} \cos \left(\frac{\pi}{n}(r-1)\left(x-\frac{1}{2}\right)\right)\right)
$$

Figure 4 a) shows the coefficients of the cosine series of the symmetric mode in a frequency spectrum. A crucial insight is that each of the terms will act as a separate grating. Two properties determine their effect on the waveguided light. First, the magnitude of each series coefficient determines the amplitude of the part of the coupling coefficient sharing the same frequency. Hence it determines the strength of the coupling. Second, the period $\Lambda_{g, r}$ of each of the terms dictate which mode will be coupled to. In the slab waveguide under question here, three candidates exist namely guided modes, cladding modes, and radiation modes. A phase matching condition is used to extract the coupled modes.

$$
2 \delta=\beta_{0}-\beta_{r}-2 \pi / \Lambda_{g, r}
$$

Here, $\delta$ is the phase matching constant, $\beta_{0}$ and $\beta_{r}$ are the propagation constant of the guided mode and new mode, respectively. Note that the negative sign of the new mode indicates that it is assumed to be propagating backwards. Strong coupling can be achieved when $\delta$ is close to zero. Only a limited number of the frequency components contribute in the coupling coefficient expansion. The few that remain require some attention as they might cause coupling to an undesired mode. The mode intervals, detailing which type of mode the new modes belong to, can be found from the wavevector and the refractive indices of the layers of the slab waveguide. Figure $4 \mathrm{~b}$ ) shows the options of the mode coupling. Since the refractive indices of the cladding, substrate and waveguide denoted as $n_{c}, n_{s}$ and $n_{f}$ are equal to $1,1.978$ and 2.488, the intervals are equal to $1.14 \times 10^{7}, 2.26 \times 10^{7}$ and $2.77 \times 10^{7}$ for $n_{c} k_{0}, n_{s} k_{0}$ and $n_{f} k_{0}$ respectively. Table 1 details the frequency, period, intensity and resulting mode of the prominent frequency components.

Table 1. Overview of the relevant frequency components found in the coupling coefficient of the symmetric mode.

\begin{tabular}{|l|l|l|c|l|}
\hline Spatial Frequency $(1 / \mathrm{nm})$ & Period $(\mathrm{nm})$ & Intensity (a.u.) & Wavevector $\beta_{r}(1 / \mathrm{m})$ & Resulting Mode \\
\hline 0.005 & 200 & 0.47176 & $-5.66 \times 10^{6}$ & Radiation Mode \\
\hline 0.025 & 40 & 0.06009 & $-1.31 \times 10^{8}$ & Cladding Mode \\
\hline 0.045 & $200 / 9$ & 0.02675 & $-2.57 \times 10^{8}$ & Guided Mode \\
\hline 0.065 & $200 / 13$ & 0.01617 & $-3.82 \times 10^{8}$ & $/$ \\
\hline
\end{tabular}

The three remaining options are now addressed. For the guided mode, a dispersion relation exists that indicates the frequency location of a wavevector mode. Here, no mode exists besides the original guided mode and this coupling term will thus not play a significant role. The cladding modes on the other hand form, like the radiation modes, a continuous spectrum unconstrained by a dispersion relation. Hence coupling to this mode can be expected, although its strength is still rather limited compared to the found radiation mode. Moreover, the mode is still confined to the structure and will act like an internal loss mechanism instead of disturbing the outcoupled signal. Again, this mode is not a real concern for the application. The radiation mode exhibits the highest intensity and is going to be analyzed separately. The outcoupling angle of the radiation mode can also be approximated here. Using $\beta_{r}, n_{f} k_{0}$ and Snell's law the outcoupling angle can be estimated to be $-30.6^{\circ}$. 

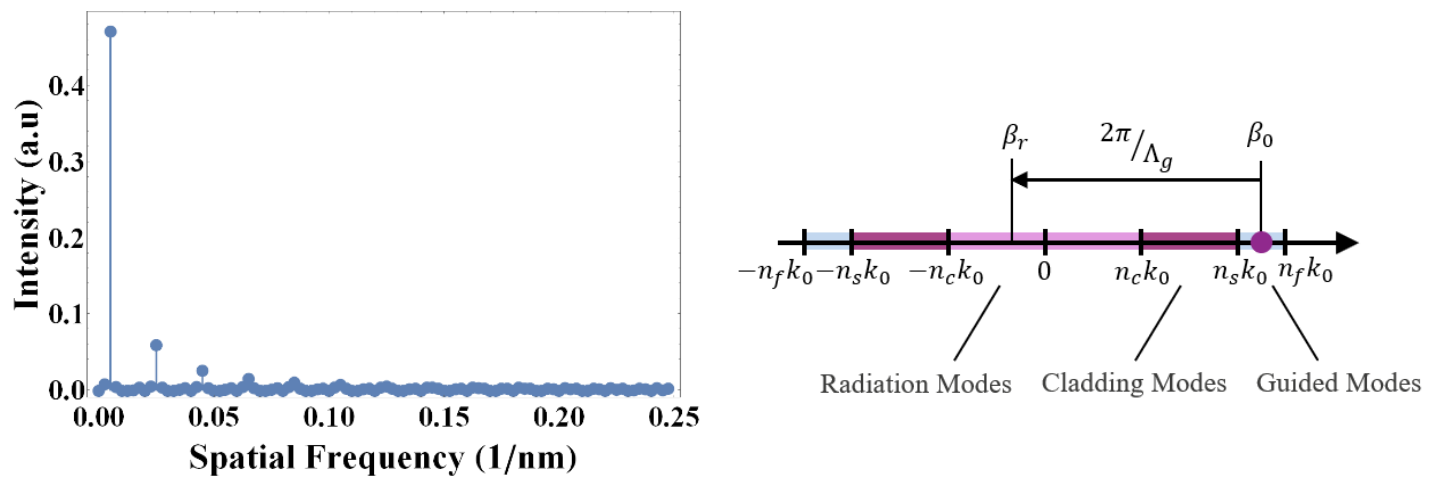

Figure 4: a) The frequency spectrum of the calculated symmetric mode coupling coefficient, for clarity the frequencies between 0.25 and 0.5 have been left out due to them being essentially zero. b) A linear scale on which the various $\mathrm{k}$ vector ranges are indicated.

\section{FINITE DIFFERENCE TIME DOMAIN SIMULATIONS}

A finite difference time domain simulation was set up using Lumerical FDTD. To incorporate the modulated waveguide, the complete permittivity tensor was imported for each of the points of the 200 by 200 sized dataset. The tensors were then used to execute a matrix transform on each separate $1 \mathrm{~nm}^{2}$ element such that the modulated refractive index mimics a realworld implementation. The single period of the modulated waveguide was subsequently copied 124 times to form a $25 \mu \mathrm{m}$ long waveguide structure. The optical and physical properties of the conducting cladding have been taken into account. To be as accurate as possible, metal contacts and a Si backplane were included at the bottom. A monitor was placed above the waveguide to capture any outcoupled light. A mode source was used to inject a TE guided mode into the structure.

The far-field characteristics of the monitor showed that only the p polarized intensity or light in the xz-plane was substantially present, indicating the expected TE to TM mode conversion. The outcoupling angle also aligns with the estimated value calculated above showing a maximum at $-29.7^{\circ}$ (Fig. 5a). Looking at the directionality plot, the highest sidelobes lay $6.80 \mathrm{~dB}$ lower than the main lobe (Fig. $5 \mathrm{~b}$ ). This result illustrates the high functionality of such a grating
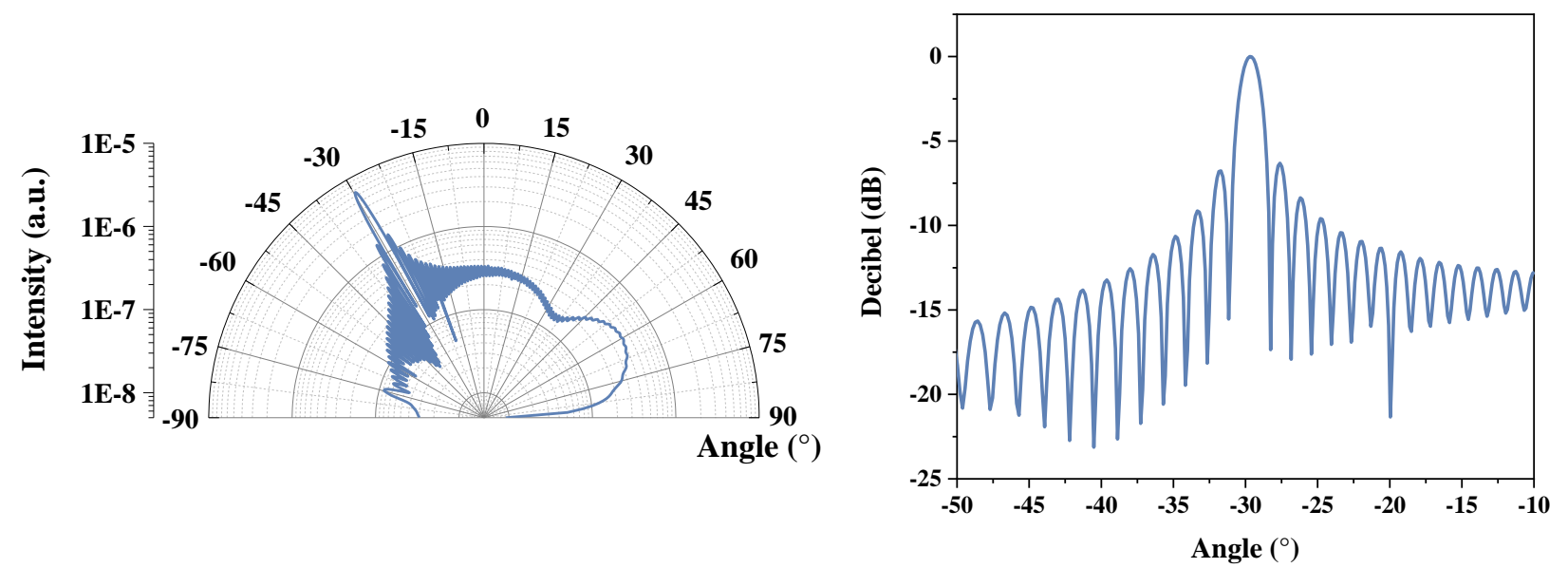

Figure 5: a) Radial plot showing the p polarized intensity coupled out of the waveguide consisting of 124 periods. b) Plot of the directionality of the outcoupled light, showing the main and side lobes on a decibel scale.

structure within a $25 \mu \mathrm{m}$ long waveguide. Further improvement can be achieved by employing a longer waveguide. The outcoupled power after $25 \mu \mathrm{m}$ is $3.48 \times 10^{-5}$ of the injected power. The analytical model outlined in Section 4 , can be used here as well. For a similarly size grating the model expects $3.64 \times 10^{-5}$ to be coupled out of the waveguide, indicating that both methods align well. 
The absolute strength of the coupling is rather low although it must be kept in mind that conservative potentials were applied to the electrodes. Additionally, the relation between the applied potential and outcoupled power is not linear. The solution of the coupled mode equations results in the coefficients $\gamma$ and $\Delta \beta$, describing the coupling loss and frequency shift of the guided mode exponential decay. Interestingly, is that the coupling loss relies on the square of the coupling coefficient, such that, tripling the potential and electric fields leads to a near a tenfold increase in the outcoupled power. Furthermore, the aspired applications have typical sizes in the centimeter range hence much longer waveguides will be implemented. Again, assuming a tripling in the electric field, the model predicts a 25 percent power output by a $2.5 \mathrm{~cm}$ long waveguide.

\section{CONCLUSION}

An electro-optically modulated grating coupler was presented that can be used as backbone for various beam steering applications. The high versatility of the proposed design allows for the generation of a wide range of gratings shaped for the optical spectrum by a dedicated spatial electrical potential setting. Our method, employing simulated electric fields, non-linear optics and coupled mode theory, can similarly be extended to any kind of diffractive optical element. Its strength lies in the use of a cosine series expansion that allows the elimination of noise and non-relevant components in the coupling behavior. In the shown example only a single component of the expansion lead to significant coupling. The mode conversion, calculated with both a theoretical approach and a Lumerical simulation, aligns well with expectations. The coupling efficiency appears rather low although conservative potentials were assumed to stay clear of dielectric breakdown.

This work has received funding from the European Research Council (ERC) under the European Union's Horizon 2020 research and innovation programme (grant agreement No 742299). G.C. has received an SB PhD fellowship of the FWO (application number 1S91520N). 


\section{REFERENCES}

[1] Smalley, D., Smithwick, Q., Bove, V. et al., "Anisotropic leaky-mode modulator for holographic video displays," Nature 498, 313-317 (2013)

[2] Lee, S.-Y. et al., "Holographic image generation with a thin-film resonance caused by chalcogenide phase-change material," Sci. Rep. 7, 41152 (2017)

[3] C. Y. H. Yong-Hae Kim, G. H. Kim, S. M. Cho, K. Choi, S. Cheon and C.-S. Hwang, "Towards 1.5 micrometer pixel pitch holographic display using Ge2Sb2Te5 phase change material," Digital Holography and 3-D Imaging, (2019)

[4] Maeno K., Fukaya N., Nishikawa O., Sato K., and Honda T. "Electro-holographic display using 15mega pixels LCD", Proc. SPIE 2652, (1996)

[5] Häussler R., Schwerdtner A., and Leister N., "Large holographic displays as an alternative to stereoscopic displays", Proc. SPIE 6803, (2008)

[6] Michalkiewicz A., Kujawinska M., Kozacki T., Wang X. and Bos P.J., "Holographic three-dimensional displays with liquid crystal on silicon spatial light modulator", Proc. SPIE 5531, (2004);

[7] Takaki Y. and Okada N., "Hologram generation by horizontal scanning of a high-speed spatial light modulator," Appl. Opt. 48, 3255-3260 (2009)

[8] Kreis T.M., Aswendt P. and Hoefling R., "Hologram reconstruction using a digital micromirror device," Opt. Eng. 40(6), 926-933 (2001)

[9] Kulishov M., Cheben P., Daxhelet X. and Delprat S., "Electro-optically induced tilted phase gratings in waveguides", J. Opt. Soc. Am. B 18(4), 457-463, (2001)

[10] Kulishov M., Daxhelet X., Gaidi M. and Chaker M., "Electronically reconfigurable superimposed waveguide long-period gratings", J. Opt. Soc. Am. A 19(8), 1632-1648, (2002)

[11] Kulishov M., Daxhelet X., Gaidi M. and Chaker M., "Transmission spectrum reconfiguration in long-period gratings electrically induced in pockels-type media with the help of a periodical electrode structure", Journal of lightwave technology 22(3), 923-933, (2004)

[12] Petraru A., Schubert J., Schmid M., and Buchal C., "Ferroelectric BaTiO3 thin-film optical waveguide modulators", Applied Physics Letters 81(8), 1375-1377, (2002)

[13] Merz W.J., "The Electric and Optical Behavior of BaTiO3 Single-Domain Crystals", Phys. Rev. 76(8), 12211225 (1949)

[14] Wemple S.H., Didomenico M. and Camlibel I., "Dielectric and optical properties of melt-grown BaTiO3”, Journal of Physics and Chemistry of Solids 29(10), 1797-1803 (1968)

[15] Piccirillo A. and Gobbi A.L., "Physical-Electrical Properties of Silicon Nitride Deposited by PECVD on III-V Semiconductors", J. Electrochem. Soc. 137(12) 3910-3917 (1990)

[16] Maeda M. and Arita Y., "Electrical properties and their thermal stability for silicon nitride films prepared by plasma-enhanced deposition", J. Appl. Phys. 53(10), 6852- 6856 (1982)

[17] R. Boyd. [Nonlinear Optics], Academic Press, Inc., USA, 511 - 542 (2008)

[18] Norton S.M, Erdogan T, and Morris G.M., "Coupled-mode theory of resonant-grating filters," J. Opt. Soc. Am. A 14(3), 629-639 (1997)

[19] Kogelnik H., [Theory of dielectric waveguides], Springer-Verlag, Berlin, 13-81 (1975)

[20]Lifante G., [Integrated Optics: Fundamentals], John Wiley \& Sons, Inc., 98-135 